\title{
Compartimentos de carbono orgânico em Latossolo cultivado com hortaliças sob diferentes manejos
}

\author{
Carlos Eduardo Pacheco Lima(1), Mariana Rodrigues Fontenelle(1), Nuno Rodrigo Madeira(1), Juscimar da Silva( ${ }^{(1)}$, \\ Ítalo Moraes Rocha Guedes ${ }^{(1)}$, Luciana Rodrigues Borba Silva ${ }^{(1)}$ e Daiane Costa Soares ${ }^{(1)}$

\begin{abstract}
(1)Embrapa Hortaliças, Rodovia BR-060, Km 09, Fazenda Tamanduá, CEP70351-970 Brasília, DF, Brasil. E-mail: carlos.pacheco-lima@embrapa.br, mariana.fontenelle@embrapa.br,nuno.madeira@embrapa.br,juscimar.silva@embrapa.br,italo.guedes@embrapa.br,daianeagro2011@gmail.com, lborba27@gmail.com
\end{abstract}

\begin{abstract}
Resumo - O objetivo deste trabalho foi avaliar os teores de carbono orgânico total (COT) e das frações granulométricas da matéria orgânica de um Latossolo Vermelho cultivado com hortaliças, sob diferentes sistemas de manejo e de cultivo de plantas de cobertura. O experimento foi realizado por seis anos, em delineamento de blocos ao caso, em arranjo fatorial $3 \times 2$, com três sistemas de manejo do solo - plantio direto (SPD), preparo reduzido (PPR) e preparo convencional (SPC) - e dois cultivos de cobertura, um de milho solteiro e outro de milho consorciado com mucuna-cinza (Stizolobium niveum) (MM), com quatro repetições. Os teores de COT, estimados para a camada $0,00-0,30 \mathrm{~m}$, foram maiores em SPD e PPR. Independentemente da profundidade avaliada, o consórcio MM promoveu os maiores teores de carbono orgânico particulado (COP), que foram fortemente influenciados pela incorporação da palhada. Em todas as camadas, observaramse correlações negativas entre os teores de COP e os de carbono orgânico associado a minerais (COAM), indício de ocorrência do efeito "priming". Os teores de COT correlacionaram-se positivamente aos de COAM, o que destaca a importância de mecanismos de estabilização da matéria orgânica no incremento dos teores de COT no solo.
\end{abstract}

Termos para indexação: carbono associado a minerais, carbono orgânico particulado, carbono orgânico total, efeito "priming", plantio direto de hortaliças, sequestro de carbono.

\section{Organic carbon pools in an Oxisol cultivated with vegetables under different managements}

\begin{abstract}
The objective of this work was to evaluate the contents of total organic carbon (COT) and of the organic matter fractions in an Oxisol cultivated with vegetables under different soil management systems and with different cover crops. The experiment was carried out for six years in a randomized block design, in a $3 \times 2$ factorial arrangement with three soil management systems - no-tillage (SPD), minimum tillage (PPR), and conventional system (SPC) -, and two cover crops, one of maize and the other of maize intercropped with Stizolobium niveum (MM), with four replicates. COT levels, estimated for $0.00-0.30-\mathrm{m}$, soil depth were higher in SPD and PPR. Regardless of the evaluated soil depth, MM intercropped system promoted higher contents of particulate organic carbon (COP), which were strongly affected by straw incorporation. In all soil depths, negative correlations were observed between the contents of COP and those of carbon associated to minerals (COAM), which indicate the occurrence of priming effect. COT contents were positively correlated to those of COAM, stressing the importance of soil organic matter stabilization mechanisms for improving COT contents in the soil.
\end{abstract}

Index terms: carbon associated to minerals, particulate organic carbon, total organic carbon, priming effect, no-tillage for vegetables, carbon sequestration.

\section{Introdução}

Para que ocorra sequestro de carbono, os sistemas de manejo devem adicionar elevadas quantidades de biomassa ao solo, causar o mínimo distúrbio em sua estrutura, além de promover a conservação de solo e da água, incrementar a atividade e a diversidade da fauna edáfica, e fortalecer os mecanismos da ciclagem de elementos (Lal, 2004). A eficiência do sistema plantio direto (SPD) como sequestrador de carbono, em solos brasileiros, tem sido mostrada por diversos trabalhos, como os de Corazza et al. (1999), Piva et al.

Pesq. agropec. bras., Brasília, v.51, n.4, p.378-387, abr. 2016

DOI: 10.1590/S0100-204X2016000400011 
(2012), Conceição et al. (2013) e Reis et al. (2014). Esse sistema apresenta, como principais fundamentos, a manutenção no solo de restos culturais de plantas durante todo o ciclo da cultura, a rotação de culturas e o revolvimento mínimo do solo.

A área cultivada com hortaliças no Brasil atinge cerca de 810 mil hectares (Anuário brasileiro de hortaliças, 2013). O plantio convencional é o sistema de manejo do solo utilizado na maior parte dos sistemas de cultivo de oleráceas no país e, geralmente, apresenta elevado aporte de insumos e intenso revolvimento do solo. Assim, o desenvolvimento de sistemas conservacionistas para o cultivo de hortaliças tem grande relevância para o Brasil, e o sistema plantio direto de hortaliças (SPDH) pode ser uma alternativa para aumentar a sustentabilidade dessa atividade.

O equilíbrio entre as frações lábeis, como o carbono orgânico particulado (COP), e as não lábeis, como o carbono orgânico associado a minerais do solo (COAM), da matéria orgânica do solo (MOS) é de suma importância para que o sequestro de carbono efetivamente ocorra em solos tropicais (Conceição et al., 2013).

A quantificação das frações granulométricas da MOS tem sido amplamente utilizada para avaliar alterações em suas frações lábeis ou estáveis, de acordo com o sistema de manejo do solo utilizado, em solos tropicais e subtropicais. Cabe ressaltar, entretanto, que a maior parte da literatura está relacionada ao SPD para grandes culturas, sobretudo grãos. Esses trabalhos comumente relatam aumento dos teores ou dos estoques de COP (Bayer et al., 2004; Costa et al., 2004; Briedis et al., 2012) e de COAM (Reis et al., 2014), com consequente aumento dos estoques de COT no solo. Alterações marcantes nessas variáveis têm sido observadas, mesmo com curto prazo de adoção do SPD, acompanhadas de melhoria da agregação do solo. No entanto, as alterações dos teores e estoques de COAM têm sido associados a manejos de mais longo prazo, acompanhadas da humificação da matéria orgânica lábil e de sua maior interação com componentes mineralógicos das frações silte e argila.

Em contrapartida, alguns trabalhos têm mostrado que o aporte de matéria orgânica fresca pode levar ao aumento das taxas de degradação da matéria orgânica estável do solo (Fontaine et al., 2007), em decorrência do efeito "priming". Esse fenômeno também pode ser potencializado por elevados aportes de $\mathrm{N}$ em ambientes ricos em C (Kuzyakov et al., 2000), o que é comum em sistemas de produção de hortaliças. Isso desperta dúvidas sobre a capacidade desses sistemas de sequestrar carbono ao longo do tempo, mesmo sob manejo conservacionista do solo.

O objetivo deste trabalho foi avaliar os teores de carbono orgânico total (COT) e das frações granulométricas da matéria orgânica de um Latossolo Vermelho cultivado com hortaliças, sob diferentes sistemas de manejo e de cultivo de plantas de cobertura.

\section{Material e Métodos}

O experimento de longa duração deste trabalho, realizado no setor de campos experimentais da Embrapa Hortaliças, na área rural do Gama, DF $\left(15^{\circ} 56^{\prime} \mathrm{S}\right.$, $48^{\circ} 08^{\prime} \mathrm{W}$, a 997,6 $\mathrm{m}$ de altitude). O clima local é do tipo Aw, conforme classificação de Köppen-Geiger.

O solo da área é um Latossolo Vermelho distrófico típico, de textura muito argilosa, cujas características químicas anteriormente à implantação do experimento estão descritas na Tabela 1. Os atributos de fertilidade, observados em cada parcela experimental, após seis anos de condução do experimento, estão descritos na Tabela 2. O experimento foi implantado no final de 2007. A área, que apresentava vegetação original de campo-sujo, no bioma Cerrado, estava em uso sob sistema convencional de produção de hortaliças desde o início da década de 1980.

Utilizou-se o delineamento de blocos ao acaso, com quatro repetições, em arranjo fatorial $3 \times 2$, com três sistemas de manejo - sistema plantio direto, (SPD), sistema de preparo reduzido com incorporação subsuperficial da palhada (PPR) e sistema de preparo convencional com incorporação profunda da palhada (SPC) - e dois cultivos de cobertura, um com milho solteiro e outro com milho consorciado com mucuna-cinza (Stizolobium niveum). As parcelas tinham $9 \mathrm{~m}$ de largura e $12 \mathrm{~m}$ de comprimento, com área total de $108 \mathrm{~m}^{2}$.

No SPD, as operações de preparo da área se resumiram à adubação e à correção do solo, bem como à formação de palhada e à abertura das covas ou linhas de plantio. No PPR, além das operações utilizadas para o sistema plantio direto, utilizou-se também um gradeamento leve, com grade niveladora semiaberta, para incorporação superficial da palhada. Por fim, no SPC utilizou-se uma aração e duas gradagens, além das 
operações referentes à adubação, correção e abertura das covas ou linhas de plantio. Cabe ressaltar que a rotação de culturas e o uso das plantas de cobertura foram as práticas conservacionistas comuns a todos os sistemas. No SPC, no entanto, houve incorporação profunda dos resíduos culturais.

O histórico de cultivo das hortaliças e das adubações realizadas foi o seguinte: ano 1, cultivo de cebola com adubação de plantio de $600 \mathrm{~kg} \mathrm{ha}^{-1}$ de $\mathrm{N}-\mathrm{P}_{2} \mathrm{O}_{5}-\mathrm{K}_{2} \mathrm{O}$ 04-30-16 e $400 \mathrm{~kg} \mathrm{ha}^{-1}$ de sulfato de amônio em cobertura; ano 2, cultivo de repolho com adubação de plantio de $1.250 \mathrm{~kg} \mathrm{ha}^{-1}$ de superfosfato simples e $250 \mathrm{~kg} \mathrm{ha}^{-1}$ de sulfato de amônio, com $400 \mathrm{~kg} \mathrm{ha}^{-1}$ de sulfato de amônio como adubação de cobertura; ano 3, cultivo de brócolis, com adubação de plantio de $1.000 \mathrm{~kg} \mathrm{ha}^{-1}$ de superfosfato simples e $250 \mathrm{~kg} \mathrm{ha}^{-1} \mathrm{de}$ sulfato de amônio, além de $500 \mathrm{~kg} \mathrm{ha}^{-1}$ de sulfato de amônio em cobertura; ano 4, cultivo de abóbora com adubação de plantio de $300 \mathrm{~kg} \mathrm{ha}^{-1}$ de superfosfato simples e $150 \mathrm{~kg} \mathrm{ha}^{-1}$ sulfato de amônio; ano 5, cultivo de repolho com adubação de plantio de $1.000 \mathrm{~kg} \mathrm{ha}^{-1}$ de superfosfato simples e $250 \mathrm{~kg} \mathrm{ha}^{-1}$ de sulfato de amônio, além de $500 \mathrm{~kg} \mathrm{ha}^{-1}$ de sulfato de amônio em cobertura; ano 6 , cultivo de brócolis, com adubação de plantio de $1.000 \mathrm{~kg} \mathrm{ha}^{-1}$ de superfosfato simples e

Tabela 1. Média \pm erro-padrão dos atributos de fertilidade do solo, anteriormente à implantação do experimento.

\begin{tabular}{|c|c|c|c|c|c|c|c|c|c|c|c|c|}
\hline \multirow[t]{2}{*}{ Camada } & \multirow[t]{2}{*}{$\mathrm{pH}$} & $P$ & K & $\mathrm{Ca}$ & $\mathrm{Mg}$ & $\mathrm{Al}$ & $\mathrm{H}^{+}$ & SB & $\mathrm{t}$ & $\mathrm{T}$ & \multirow{2}{*}{$\begin{array}{c}\mathrm{V} \\
(\%)\end{array}$} & \multirow{2}{*}{$\begin{array}{c}\text { COT } \\
\left(\mathrm{g} \mathrm{kg}^{-1}\right)\end{array}$} \\
\hline & & \multicolumn{2}{|c|}{ 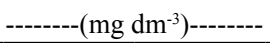 } & & & & & & & & & \\
\hline 00 & & $76 \pm 17$ & $254 \pm 8$ & 12 , & $3,7 \pm 0$ & & 5,4 & 0 & 17. & $22,3 \pm 0,5$ & $5,5 \pm 2,8$ & $17,3 \pm 0,7$ \\
\hline 0 & $3 \pm$ & & $94 \pm 6$ & & & & & & & & & $12,0 \pm 1,2$ \\
\hline $00-0,30 \mathrm{~m}$ & $, 7 \pm 0,1$ & $60 \pm 15$ & $230 \pm 26$ & $11,6 \pm 0,7$ & $3,7 \pm 0,0$ & $0,1 \pm 0,0$ & $5,5 \pm 0,3$ & $15,9 \pm 0,7$ & $15,9 \pm 0,7$ & $21,4 \pm 0,7$ & $73,9 \pm 1,4$ & $16,3 \pm 1,2$ \\
\hline
\end{tabular}

Tabela 2. Média \pm erro-padrão dos atributos de fertilidade do solo, após seis ciclos de produção de hortaliças.

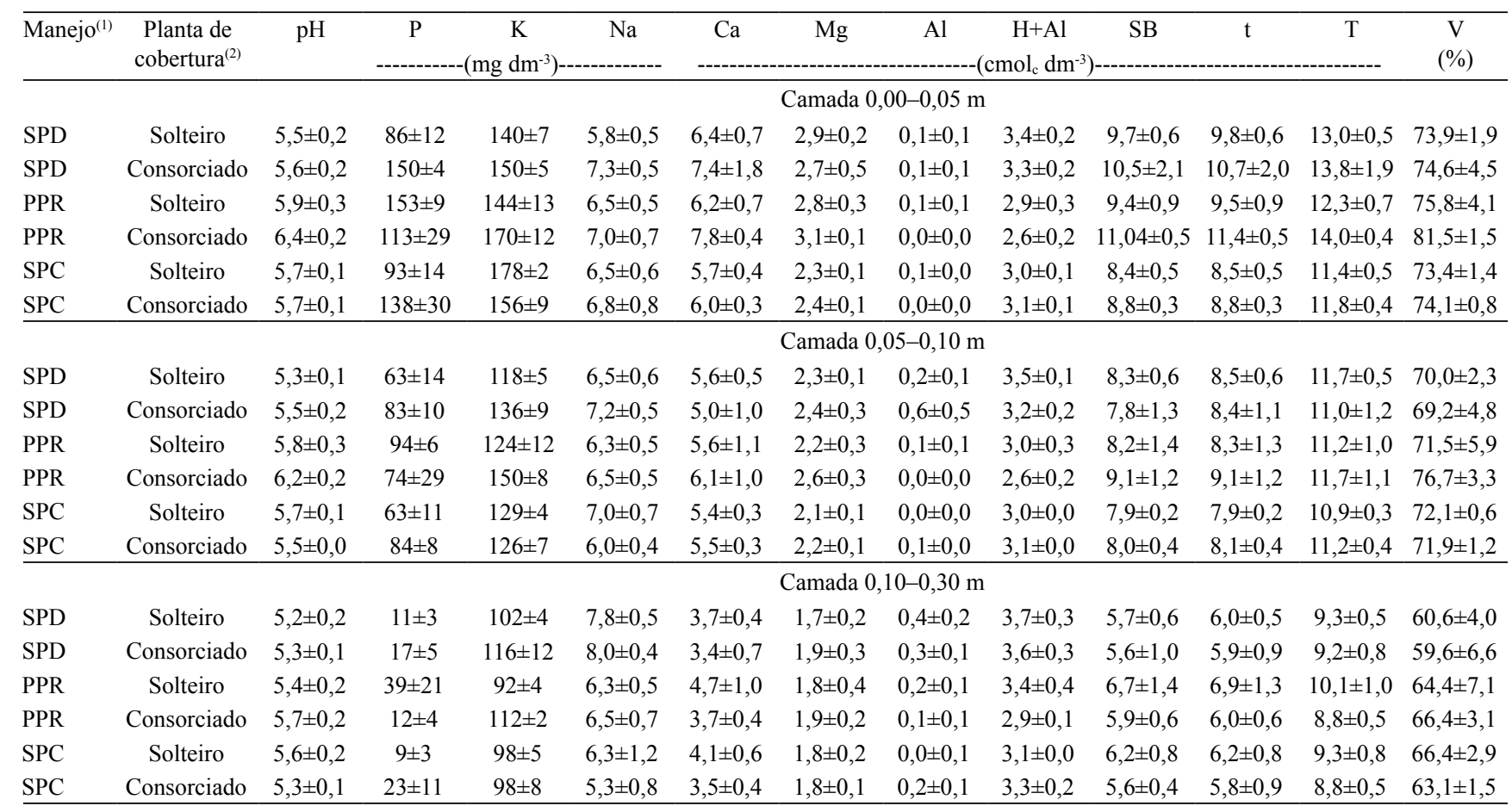

${ }^{(1)} \mathrm{SPD}$, sistema plantio direto; PPR, sistema de preparo reduzido; e SPC, sistema de plantio convencional. ${ }^{(2)}$ Milho solteiro ou milho cultivado em consórcio com mucuna-cinza. 
$250 \mathrm{~kg} \mathrm{ha}^{-1}$ de sulfato de amônio, além de $500 \mathrm{~kg} \mathrm{ha}^{-1}$ de sulfato de amônio em cobertura.

A formação da palhada para cobertura dos solos, nos tratamentos onde o manejo utilizado foi o SPD, e para rotação de culturas no PPR e no SPC, foi realizada da seguinte forma: a semeadura das plantas de cobertura foi efetuada no período chuvoso, entre novembro e dezembro, e o plantio das hortaliças, entre os meses de fevereiro e maio. A semeadura do milho foi realizada em espaçamento entre linhas de $0,80 \mathrm{~m}$, com cinco sementes por metro e $100 \mathrm{~kg} \mathrm{ha}^{-1}$ de $\mathrm{P}_{2} \mathrm{O}_{5}$. Utilizou-se o híbrido comercial AG $1045 \mathrm{em}$ estande de 55 mil plantas por hectare. A mucuna-cinza foi semeada em espaçamento entre linhas de 1,60 m e duas sementes por metro, 30 dias após semeadura do milho. As palhadas foram formadas a partir do uso de um triturador-desintegrador em plantas com cerca de 50 dias de idade. Na rebrota, utilizou-se glifosato e, cerca de uma semana antes do plantio da hortaliça, o Paraquat, de acordo com as recomendações dos fabricantes.

Coletadas em minitrincheiras de $0,8 \times 0,8 \mathrm{~m}$, com $0,5 \mathrm{~m}$ de profundidade, as amostras foram extraídas das camadas de $0-0,05,0,05-0,10$ e $0,10-0,30 \mathrm{~m}$ e, em seguida, foram secas ao ar e passadas em peneira com malha de $2 \mathrm{~mm}$.

A determinação dos teores de COT, bem como o de suas frações granulométricas, foi realizada conforme o método Walkley-Black. O fracionamento da matéria orgânica foi realizado conforme Cambardella \& Elliott (1992). Para tal, $20 \mathrm{~g}$ de solo (terra fina seca ao ar) foram colocados em frascos de centrífuga, aos quais foram acrescentados $60 \mathrm{~mL}$ de hexametafosfato de sódio, antes da agitação horizontal, a $200 \mathrm{rpm}$, por 15 horas. $\mathrm{O}$ conteúdo retido em peneira de malha de $53 \mu \mathrm{m}$ foi lavado, seco em estufa a $40^{\circ} \mathrm{C}$ e pesado, e os teores de COT foram, então, determinados. Os teores de COP foram determinados após correção de acordo com a textura dos solos, conforme a seguinte equação: $\mathrm{COP}=\mathrm{COT}(>53 \mu \mathrm{m}) \times \mathrm{m}(>53 \mu \mathrm{m}) / 100$; em que: $\operatorname{COT}(>53 \mu \mathrm{m})$ é o teor de COT no material retido em peneira de malha superior a $53 \mu \mathrm{m}$; e m é a percentagem, em massa, da TFSA retida nessa mesma peneira.

Os teores de COAM foram quantificados pela diferença entre os teores de COT e COP. Por fim, os teores de COP, COAM e COT, acumulados nos diferentes sistemas até a profundidade de $0,30 \mathrm{~m}$, foram calculados a partir da média ponderada de seus teores, como $\mathrm{Cx}_{0,00-0,30}=5\left(\mathrm{Cx}_{0,00-0,05}\right)+5\left(\mathrm{Cx}_{0,05-0,10}\right)+$ $20\left(\mathrm{Cx}_{0,10-0,30}\right) / 30$, em que $\mathrm{x}$ representa o tipo de carbono avaliado (COP, COAM ou COT).

Antes de serem submetidos à análise de variância, os dados tiveram sua distribuição normal verificada. As médias foram agrupadas pelo teste de Scott-Knott, a $5 \%$ de probabilidade. As relações dos atributos de fertilidade e das frações orgânicas com os teores de COT foram verificadas por meio da avaliação de correlações de Pearson.

\section{Resultados e Discussão}

Nas diferentes camadas, não se observou interação entre os fatores, para todas as variáveis analisadas, com exceção da interação significativa entre os teores de COP e COAM, na camada de 0,05-0,10 m (Tabela 3).

Os sistemas SPD e o PPR foram os mais eficientes em acumular COT na camada de 0,00-0,30 m (Tabela 4). Após seis anos de adoção, o SPD foi capaz de aumentar em cerca de $15 \%$ seus teores de COT; enquanto o PPR

Tabela 3. Análise de variância resumida dos teores de carbono orgânico particulado (COP), carbono orgânico associado a minerais (COAM) e carbono orgânico total (COT), nas quatro profundidades avaliadas em um Latossolo Vermelho cultivado por seis anos com hortaliças, nos sistemas de manejo plantio direto, preparo reduzido e plantio convencional, com uso de milho solteiro ou consorciado com mucuna-cinza como plantas de cobertura.

\begin{tabular}{|c|c|c|c|c|c|c|}
\hline \multirow{2}{*}{$\begin{array}{l}\text { Fonte de } \\
\text { variação }\end{array}$} & \multicolumn{2}{|c|}{ COP } & \multicolumn{2}{|c|}{ COAM } & \multicolumn{2}{|c|}{ COT } \\
\hline & QMR & $\mathrm{Fc}$ & QMR & $\mathrm{Fc}$ & QMR & $\mathrm{Fc}$ \\
\hline & \multicolumn{6}{|c|}{ Camada $0,00-0,05 \mathrm{~m}$} \\
\hline Sistemas de manejo & 133,85 & 52,26 & 254,60 & 25,48 & 186,47 & 17,67 \\
\hline Plantas de cobertura & 27,09 & 10,58 & 13,44 & 1,35 & 2,34 & 0,22 \\
\hline \multirow[t]{2}{*}{ Interação } & 5,52 & 2,16 & 0,62 & 0,06 & 2,44 & 0,23 \\
\hline & \multicolumn{6}{|c|}{ Camada $0,05-0,10 \mathrm{~m}$} \\
\hline Sistemas de manejo & 98,73 & 30,40 & 165,82 & 33,54 & 24,77 & 5,92 \\
\hline Plantas de cobertura & 77,40 & 23,76 & 62,76 & 12,69 & 0,77 & 0,18 \\
\hline \multirow[t]{2}{*}{ Interação } & 12,23 & 3,76 & 19,59 & 3,96 & 1,91 & 0,46 \\
\hline & \multicolumn{6}{|c|}{ Camada $0,10-0,30 \mathrm{~m}$} \\
\hline Sistemas de manejo & 43,89 & 50,64 & 63,01 & 18,13 & 1,15 & 0,32 \\
\hline Plantas de cobertura & 3,46 & 3,99 & 4,38 & 1,26 & 1,37 & 0,38 \\
\hline \multirow[t]{2}{*}{ Interação } & 1,81 & 2,09 & 5,01 & 1,44 & 0,40 & 0,11 \\
\hline & \multicolumn{6}{|c|}{ Camada $0,00-0,30 \mathrm{~m}$} \\
\hline Sistemas de manejo & 15,97 & 33,40 & 58,77 & 19,50 & 14,78 & 4,51 \\
\hline Plantas de cobertura & 1,35 & 2,82 & 0,29 & 0,09 & 0,40 & 0,12 \\
\hline Interação & 0,02 & 0,04 & 2,73 & 0,90 & 2,31 & 0,70 \\
\hline
\end{tabular}

Pesq. agropec. bras., Brasília, v.51, n.4, p.378-387, abr. 2016 DOI: 10.1590/S0100-204X2016000400011 
foi capaz de aumentá-los em cerca de $10 \%$, e o SPC em cerca de 5\%, em comparação aos teores iniciais antes da implantação do experimento. $\mathrm{O}$ incremento dos teores de $\mathrm{C}$, em sistema com revolvimento reduzido do solo, especialmente no sistema plantio direto, tem sido relatado em diversos trabalhos e está associado, entre outros fatores, ao aumento do aporte de material vegetal ao solo, ao manejo das plantas de cobertura (Gonçalves \& Ceretta, 1999; Amado et al., 2001; Bayer et al., 2003), à melhoria da qualidade física do solo (Costa et al., 2004; Wendling et al., 2005) e ao aumento da humificação da MOS (Souza \& Melo, 2003; Fontana et al., 2006).

O SPD foi ainda o sistema de manejo com os maiores teores de COAM na camada 0,00-0,30, seguido do PPR e, por fim, do SPC. Além disso, o SPD apresentou os menores teores de COP, enquanto PPR e SPC mantiveram os maiores. Esse resultado pode estar relacionado à incorporação dos resíduos vegetais nos sistemas com revolvimento de solo, que os distribuem em profundidade. Conceição et al. (2013) concluíram que os maiores teores de COT foram observados no sistema plantio direto, e que a maior parte do C estava na forma de COAM, o que evidencia a importância dessa fração para a promoção do aumento dos teores de COT em solos tropicais e subtropicais. Cabe ressaltar também que, à medida que a estrutura do solo é fortalecida pela adoção de sistemas de manejo com baixo revolvimento, os teores de COP devem ser incrementados na forma de COP oclusa em agregados estáveis (Conceição et al., 2013). O aumento dos teores de COP livre antecedem a formação de COP oclusa em macro e microagregados; por fim, há a formação de COAM (Golchin et al., 1994). Portanto, a estabilização da MOS em longo prazo se dá pelo aumento dos teores de COAM.

O SPD foi o sistema com os maiores teores de COP na camada $0,00-0,05 \mathrm{~m}$, enquanto o PPR apresentou

Tabela 4. Teores $\left(\mathrm{g} \mathrm{kg}^{-1}\right)$ de carbono orgânico particulado (COP), carbono orgânico associado a minerais (COAM) e carbono orgânico total (COT), nas camadas $0,00-0,05 \mathrm{~m}, 0,05-0,10 \mathrm{~m}, 0,10-0,30 \mathrm{~m}$ e $0,00-0,30 \mathrm{~m}$ de um Latossolo Vermelho cultivado por seis anos com hortaliças, sob os sistemas de manejo de plantio direto (SPD), preparo reduzido (PPR) e plantio convencional (SPC), com uso de milho solteiro ou consorciado com mucuna-cinza, como plantas de cobertura ${ }^{(1)}$.

\begin{tabular}{|c|c|c|c|c|c|c|c|c|c|c|c|c|}
\hline \multirow{2}{*}{$\begin{array}{l}\text { Fator de } \\
\text { variação }\end{array}$} & \multicolumn{3}{|c|}{$0,00-0,05 \mathrm{~m}$} & \multicolumn{3}{|c|}{$0,05-0,10 \mathrm{~m}$} & \multicolumn{3}{|c|}{$0,10-0,30 \mathrm{~m}$} & \multicolumn{3}{|c|}{$0,00-0,30 \mathrm{~m}$} \\
\hline & COP & COAM & COT & COP & COAM & $\mathrm{COT}$ & COP & COAM & $\mathrm{COT}$ & COP & COAM & COT \\
\hline & \multicolumn{12}{|c|}{ Sistemas de manejo } \\
\hline SPD & $6,48 \mathrm{a}$ & $20,55 b$ & $27,03 \mathrm{a}$ & - & - & $20,68 \mathrm{a}$ & $1,02 b$ & $15,16 \mathrm{a}$ & $16,19^{\mathrm{ns}}$ & $2,14 b$ & $16,59 a$ & $18,75 \mathrm{a}$ \\
\hline PPR & $1,72 \mathrm{c}$ & $23,79 a$ & $25,53 \mathrm{a}$ & - & - & $19,36 b$ & $3,14 \mathrm{a}$ & $12,67 \mathrm{~b}$ & $15,81^{\mathrm{ns}}$ & $3,43 \mathrm{a}$ & $14,59 b$ & $18,02 \mathrm{a}$ \\
\hline \multirow[t]{2}{*}{$\underline{\mathrm{SPC}}$} & $4,36 \mathrm{~b}$ & $17,24 \mathrm{c}$ & $21,61 b$ & - & - & $18,67 \mathrm{~b}$ & $3,57 \mathrm{a}$ & $12,11 \mathrm{~b}$ & $15,68^{\mathrm{ns}}$ & $3,67 \mathrm{a}$ & $13,50 \mathrm{c}$ & $17,17 \mathrm{~b}$ \\
\hline & \multicolumn{12}{|c|}{ Plantas de Cobertura } \\
\hline M & $3,58 b$ & $20,96^{\mathrm{ns}}$ & $24,54^{\mathrm{ns}}$ & - & - & $19,47^{\mathrm{ns}}$ & $2,79^{\mathrm{ns}}$ & $13,07^{\mathrm{ns}}$ & $15,85^{\mathrm{ns}}$ & $2,94^{\mathrm{ns}}$ & $14,96^{\mathrm{ns}}$ & $17,91^{\mathrm{ns}}$ \\
\hline \multirow[t]{2}{*}{ MM } & $4,81 \mathrm{a}$ & $20,09^{\mathrm{ns}}$ & $24,90^{\mathrm{ns}}$ & - & - & $19,68^{\mathrm{ns}}$ & $2,37^{\mathrm{ns}}$ & $13,51^{\mathrm{ns}}$ & $15,94^{\mathrm{ns}}$ & $3,22^{\text {ns }}$ & $14,83^{\text {ns }}$ & $18,06^{\mathrm{ns}}$ \\
\hline & \multicolumn{12}{|c|}{ Interação entre sistemas de manejo e plantas de cobertura } \\
\hline SPD x M & - & - & - & $2,05 b$ & $18,26 \mathrm{a}$ & - & - & - & - & - & - & - \\
\hline PPR x M & - & - & - & $5,10 \mathrm{a}$ & $14,45 b$ & - & - & - & - & - & - & - \\
\hline$\underline{\mathrm{SPC} \times \mathrm{M}}$ & - & - & - & $1,68 \mathrm{~b}$ & $16,86 a$ & - & - & - & - & - & - & - \\
\hline SPD x MM & - & - & - & $2,59 \mathrm{c}$ & $18,46 \mathrm{a}$ & - & - & - & - & - & - & - \\
\hline PPR x MM & - & - & - & $7,41 \mathrm{a}$ & $11,89 \mathrm{c}$ & - & - & - & - & - & - & - \\
\hline \multirow[t]{2}{*}{$\underline{\mathrm{SPC}} \times \mathrm{MM}$} & - & - & - & $5,07 \mathrm{~b}$ & $13,57 \mathrm{~b}$ & - & - & - & - & - & - & - \\
\hline & \multicolumn{12}{|c|}{ Interação dentro de cada sistema de manejo } \\
\hline M x SPD & - & - & - & $2,09^{\mathrm{ns}}$ & $18,26^{\mathrm{ns}}$ & - & - & - & - & - & - & - \\
\hline MM x SPD & - & - & - & $2,59^{\mathrm{ns}}$ & $18,46^{\mathrm{ns}}$ & - & - & - & - & - & - & - \\
\hline M x PPR & - & - & - & $5,10 \mathrm{~b}$ & $14,45 \mathrm{a}$ & - & - & - & - & - & - & - \\
\hline MM x PPR & - & - & - & $7,41 \mathrm{a}$ & $11,89 \mathrm{~b}$ & - & - & - & - & - & - & - \\
\hline M x SPC & - & - & - & $1,68 b$ & $16,86 \mathrm{a}$ & - & - & - & - & - & - & - \\
\hline MM x SPC & - & - & - & $5,07 \mathrm{a}$ & $13,57 \mathrm{~b}$ & - & - & - & - & - & - & - \\
\hline $\mathrm{CV}(\%)$ & 22,14 & 8,93 & 7,62 & 26,29 & 8,54 & 6,06 & 21,25 & 8,12 & 6,95 & 13,02 & 6,76 & 5,84 \\
\hline
\end{tabular}

${ }^{(1)}$ Médias seguidas de letras iguais não diferem, pelo teste de Scott-Knott, a $5 \%$ de probabilidade. 
os maiores teores dessa fração na camada $0,05-0,10 \mathrm{~m}$ e, em conjunto com o SPC, na camada $0,10-0,30 \mathrm{~m}$ (Tabela 4). Esses resultados estão ligados à forma de distribuição da palhada no perfil do solo, nos diferentes sistemas. No SPD, a palhada é mantida em superfície; no PPR, é incorporada com grade semiaberta regulada para distribuí-la à profundidade aproximada de 0,10 m; e, no SPC, ela é incorporada em profundidade pelas operações de aração e gradagem, normalmente efetuadas para atingir profundidade em torno de $0,20 \mathrm{~m}$. A importância da forma do aporte da biomassa de plantas de cobertura ao solo, para o acréscimo da fração particulada da matéria orgânica, já foi relatada anteriormente (Dieckow et al., 2005; Sá \& Lal, 2009; Salvo et al., 2010; Briedis et al., 2012).

Os teores de COAM, em geral, apresentaram comportamento inverso aos de COP (Tabela 4). Assim, os maiores teores de COAM foram observados no PPR, na camada $0,00-0,05 \mathrm{~m}$, e no SPD, na camada 0,10-0,30 m. Na camada $0,05-0,10 \mathrm{~m}$, entretanto, a manutenção dos teores de COAM, nos diferentes sistemas de manejo, dependeu também do tipo cultivo das plantas de cobertura. Assim, com o uso do milho solteiro, os maiores teores de COAM foram observados no SPD e no SPC; enquanto o uso do consórcio milho e mucuna-cinza proporcionou os maiores teores dessa fração apenas no SPD.
A forte relação inversa entre os teores de COAM e os de COP (Tabela 5) indica que o aporte de matéria orgânica lábil ao solo pode promover a degradação de parte da matéria orgânica já estabilizada, em razão do efeito "priming" (Dalenberg \& Jager, 1989; Fontaine et al., 2003). Esse fenômeno de degradação de matéria orgânica estável, quando do aporte de matéria orgânica fresca, já foi relatado em vários estudos (Bell et al., 2003; Fontaine et al., 2007).

Uma das mais comuns explicações para a ocorrência do efeito "priming", após a adição de substratos com elevada relação $\mathrm{C} / \mathrm{N}$, é que os microrganismos buscam $\mathrm{N}$ na $\mathrm{MOS}$ (C/N relativamente baixa) e isso aumenta sua decomposição e a evolução de $\mathrm{CO}_{2}$ (Kuzyakov, 2010). Entretanto, as causas para a ocorrência do efeito são diversas (Kuzyakov et al., 2000; Fontaine et al., 2003). Cabe ressaltar, entretanto, que o SPD de hortaliças resultou em forte incremento do C orgânico no solo, conforme mostrado anteriormente, e que solos com cultivo de hortaliças recebem forte aporte de adubos nitrogenados, o que também pode ser uma explicação para a ocorrência do efeito "priming". De fato, os autores citados na discussão desse efeito alegam que a adição de fertilizantes minerais nitrogenados também pode levar à aceleração dos processos de mineralização da MOS.

Tabela 5. Coeficientes de correlação de Pearson entre atributos de fertilidade e frações granulométricas da matéria orgânica do solo, nas camadas 0,00-0,05 m, 0,05-0,10 $\mathrm{m}$ e $0,10-0,30 \mathrm{~m}$ de um Latossolo Vermelho cultivado com hortaliça por seis anos, sob diferentes sistemas de manejo e plantas de cobertura.

\begin{tabular}{|c|c|c|c|c|c|c|c|c|c|}
\hline \multirow[t]{2}{*}{ Atributo } & \multicolumn{3}{|c|}{$0,00-0,05 \mathrm{~m}$} & \multicolumn{3}{|c|}{$0,05-0,10 \mathrm{~m}$} & \multicolumn{3}{|c|}{$0,10-0,30 \mathrm{~m}$} \\
\hline & $\mathrm{COP}$ & COAM & COT & $\mathrm{COP}$ & COAM & $\mathrm{COT}$ & $\mathrm{COP}$ & COAM & $\mathrm{COT}$ \\
\hline$\overline{\mathrm{pH}}$ & $-0,40$ & 0,27 & $-0,04$ & 0,37 & $-0,44^{*}$ & $-0,27$ & 0,28 & $-0,23$ & $-0,04$ \\
\hline $\mathrm{P}$ & $-0,00$ & 0,15 & 0,17 & 0,31 & $-0,29$ & $-0,07$ & 0,09 & $-0,02$ & 0,10 \\
\hline $\mathrm{K}$ & $-0,29$ & $-0,24$ & $-0,51^{*}$ & 0,33 & $-0,28$ & $-0,01$ & $-0,37$ & 0,35 & 0,13 \\
\hline $\mathrm{Na}$ & 0,07 & $-0,17$ & $-0,13$ & $-0,18$ & 0,26 & 0,23 & $-0,60 *$ & $0,51^{*}$ & 0,13 \\
\hline $\mathrm{Ca}$ & $-0,07$ & 0,31 & 0,29 & 0,18 & $-0,19$ & $-0,08$ & 0,12 & 0,00 & 0,15 \\
\hline $\mathrm{Mg}$ & $-0,13$ & 0,39 & 0,33 & 0,15 & $-0,11$ & 0,03 & $-0,06$ & 0,10 & 0,08 \\
\hline $\mathrm{Al}$ & 0,19 & $-0,01$ & 0,15 & $-0,26$ & 0,36 & 0,27 & $-0,30$ & 0,20 & $-0,02$ \\
\hline $\mathrm{H}+\mathrm{Al}$ & 0,35 & $-0,15$ & 0,13 & $-0,33$ & $0,47 *$ & 0,40 & $-0,34$ & 0,24 & $-0,01$ \\
\hline $\mathrm{SB}$ & $-0,10$ & 0,35 & 0,31 & 0,18 & $-0,18$ & $-0,06$ & 0,06 & 0,04 & 0,14 \\
\hline $\mathrm{t}$ & $-0,09$ & 0,37 & 0,33 & 0,13 & $-0,11$ & 0,01 & 0,02 & 0,08 & 0,15 \\
\hline $\mathrm{T}$ & $-0,03$ & 0,36 & 0,37 & 0,12 & $-0,08$ & 0,04 & $-0,06$ & 0,15 & 0,17 \\
\hline V & $-0,23$ & 0,29 & 0,13 & 0,22 & $-0,31$ & $-0,25$ & 0,21 & $-0,09$ & 0,10 \\
\hline COP & 1,00 & $-0,50^{*}$ & 0,29 & 1,00 & $-0,86^{*}$ & $-0,06$ & 1,00 & $-0,76^{*}$ & $-0,06$ \\
\hline COAM & - & 1,00 & $0,68^{*}$ & - & 1,00 & $0,56 *$ & - & 1,00 & $0,69 *$ \\
\hline $\mathrm{COT}$ & - & - & 1,00 & - & - & 1,00 & - & - & 1,00 \\
\hline
\end{tabular}

*Significativo a 5\% de probabilidade. COP, carbono orgânico particulado; COAM, carbono orgânico associado a minerais; e COT, carbono orgânico total. 
Os teores de COT nas diferentes profundidades foram maiores em SPD e PPR, na camada 0,00$0,05 \mathrm{~m}$ e, em SPD, apenas na camada $0,05-0,10 \mathrm{~m}$. Na camada $0,10-0,30 \mathrm{~m}$, entretanto, não se observou efeito dos sistemas de manejo sobre o atributo. $\mathrm{O}$ maior teor de COT na camada superficial, em SPD, indica que, embora o efeito "priming" possa ter ocorrido (correlação negativa entre COP e COAM), a intensidade dele não foi suficiente para que o sistema perdesse sua capacidade de acumular $\mathrm{C}$ no solo. Além disso Kuzyakov (2010) sugere que a ocorrência do efeito "priming" não diminui, necessariamente, os estoques de $\mathrm{C}$ no solo, uma vez que a maioria dos estudos mostra incremento líquido dos estoques de $\mathrm{C}$ mesmo quando o fenômeno ocorre, no caso de acréscimo em COP mais significativo do que o decréscimo em COAM.

$\mathrm{O}$ consórcio milho e mucuna-cinza resultou em maiores teores de COP na camada de 0,00-0,05 m. Entretanto, na camada $0,05-0,10 \mathrm{~m}$, o consórcio somente favoreceu esse atributo nos sistemas com revolvimento de solo, PPR e SPC. Os maiores teores de COP com o consórcio pode ser explicado pelo fato de os restos culturais da mucuna-cinza serem menos suscetíveis à degradação que os do milho, o que faria com que permanecessem mais tempo no solo, na forma de COP. Carvalho et al. (2008) mostraram que a mucuna-cinza apresenta uma das menores taxas de decomposição nos sistemas convencional e plantio direto. Essas baixas taxas de decomposição têm sido atribuídas a altos teores de compostos aromáticos nos resíduos da leguminosa (Carvalho et al., 2014). A ausência de significância na camada de 0,05-0,10 m, quando do desdobramento da interação plantas de cobertura e sistema de plantio direto, sugere ainda a influência da incorporação mecânica da palhada sobre os incrementos observados no PPR e no SPC nessa camada.

Os teores de COAM, por sua vez, foram superiores nos tratamentos com milho solteiro, nas camadas 0,00 $0,05 \mathrm{~m}$ e $0,05-0,10 \mathrm{~m}$. A degradação de frações lábeis da matéria orgânica precede a humificação, que é um importante processo de estabilização da MOS (Dick et al., 2009). Assim, a presença de resíduos vegetais de mais fácil degradação, provenientes da palhada de milho, pode ter acelerado a formação de matéria orgânica não lábil, o que justificaria a manutenção dos maiores teores de COAM, com o uso do milho solteiro, nessas camadas. Destaca-se novamente a ausência de significância na camada de $0,05-0,10 \mathrm{~m}$ para $\mathrm{o}$ desdobramento plantas de cobertura dentro do sistema de plantio direto, o que reforça a percepção de que os efeitos nessa camada estão ligados ao aporte mecânico dos restos culturais das plantas de cobertura.

Os três sistemas de manejo apresentaram tendência de decréscimo nos teores COAM e COT, com a profundidade (Figura 1). O SPD também apresentou essa tendência nos teores de COP. No PPR, contudo, os teores dessa fração foram aumentados em subsuperfície $(0,05-0,10 \mathrm{~m})$ e, no SPC, o mesmo foi observado na camada $0,10-0,30 \mathrm{~m}$.

Quanto às plantas de cobertura, o uso do milho resultou em tendência de decréscimo dos teores das duas frações e do COT, com a profundidade. Com o consórcio, no entanto, observou-se tendência de acréscimo dos teores de COP, em subsuperfície, e de decréscimo de COAM e COT, em profundidade.

Os atributos de fertilidade, em geral, não se correlacionaram aos de matéria orgânica (Tabela 5). Entre esses atributos, observou-se que os teores de $\mathrm{K}$ correlacionaram-se negativamente aos de COT, na camada $0,00-0,05 \mathrm{~m}$. Na camada $0,05-0,10 \mathrm{~m}$, correlações negativas foram encontradas entre $\mathrm{pH}$ e os teores de COAM, além de correlações positivas entre $\mathrm{H}+\mathrm{Al}$ e essa fração. Na camada $0,10-0,30 \mathrm{~m}$, observaram-se correlações negativas entre os teores de COP e de $\mathrm{Na}$, e positivas entre os de COAM e Na. Apesar da significância dessas correlações, os coeficientes encontrados foram relativamente baixos. A calagem e fertilização intensas para o cultivo de hortaliças podem ter mascarado as relações dos atributos de fertilidade com os da MOS. Também é possível que o tempo de condução do experimento - seis anos - não tenha sido suficiente para que as relações, normalmente encontradas entre esses atributos, tenham se manifestado.

Uma relação que merece destaque foi a apresentada pelos teores de COAM e COT, que foi positiva nas três profundidades avaliadas. Esse resultado indica que, nas condições edafoclimáticas em que o experimento tem sido conduzido, o acúmulo de COT depende do incremento dos teores de COAM. Portanto, é possível inferir que a estabilização da MOS é fundamental para o aumento dos teores de COT. Conceição et al. (2013) verificaram que a maior parte do $\mathrm{C}$ acumulado em um Latossolo subtropical estava na forma associada aos minerais, o que reforça os resultados encontrados no presente trabalho. 

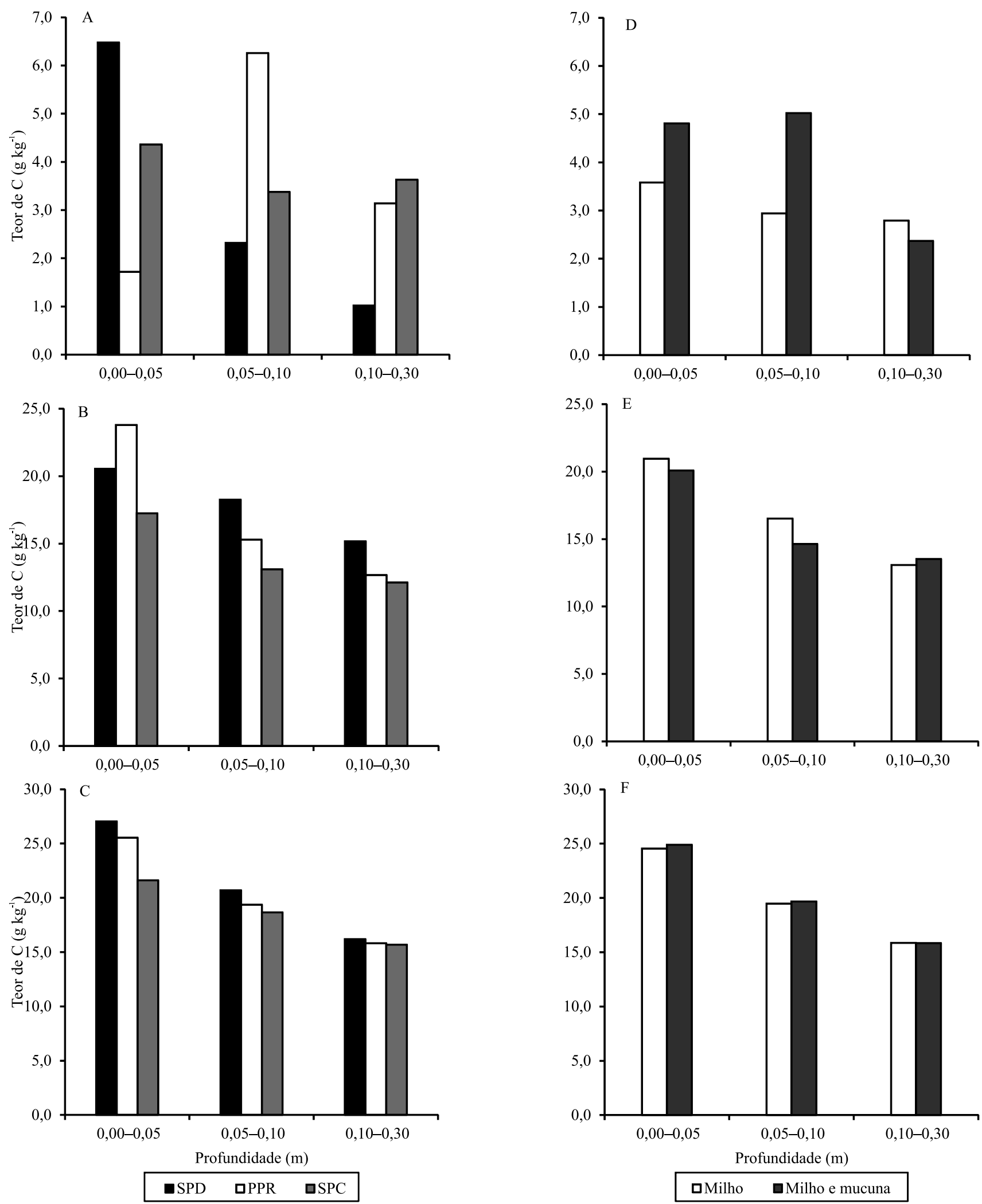

Figura 1. Teores médios de carbono orgânico particulado (COP, A e B), carbono orgânico associado a minerais (COAM, C e D) e carbono orgânico total (COT, E e F), em função dos sistemas de manejo plantio direto (SPD), preparo reduzido (PPR) e plantio convencional (SPC), com uso de milho solteiro ou consorciado com mucuna-cinza como planta de cobertura. 


\section{Conclusões}

1. Os sistemas plantio direto e de preparo reduzido são mais eficientes em acumular $\mathrm{C}$ na camada do solo de $0,00-0,30 \mathrm{~m}$, em cultivos de hortaliças.

2. Os teores de carbono orgânico particulado (COP) sofrem forte influência da incorporação dos resíduos das plantas de cobertura.

3. A estabilização da matéria orgânica na forma de carbono orgânico associado a minerais (COAM) é importante para a manutenção de maiores teores de carbono orgânico total no solo.

4. As correlações significativas e negativas entre COP e COAM, nas três camadas avaliadas, indicam a possível ocorrência do efeito "priming", possivelmente relacionado à incorporação de matéria orgânica lábil ao solo.

\section{Referências}

AMADO, T.J.C.; BAYER, C.; ELTZ, F.L.F.; BRUM, A.C.R. Potencial de culturas de cobertura em acumular carbono e nitrogênio no solo no plantio direto e a melhoria da qualidade ambiental. Revista Brasileira de Ciência do Solo, v.25, p.189-197, 2001. DOI: $10.1590 / \mathrm{S} 0100-06832001000100020$.

ANUÁRIO brasileiro de hortaliças. Santa Cruz do Sul: Gazeta, 2013. 88p.

BAYER, C.; MARTIN-NETO, L.; MIELNICZUK, J.; PAVINATO, A. Armazenamento de carbono em frações lábeis da matéria orgânica de um Latossolo Vermelho sob plantio direto. Pesquisa Agropecuária Brasileira, v.39, p.677-683, 2004. DOI: 10.1590/ S0100-204X2004000700009.

BAYER, C.; SPAGNOLLO, E.; WILDNER, L. do P.; ERNANI, P.R.; ALBURQUERQUE, J.A. Incremento de carbono e nitrogênio num latossolo pelo uso de plantas estivais para cobertura do solo. Ciência Rural, v.33, p.469-475, 2003. DOI: 10.1590/ S0103-84782003000300012.

BELL, J.M.; SMITH, J.L.; BAILEY, V.L.; BOLTON, H. Priming effect and $\mathrm{C}$ storage in semi-arid no-till spring crop rotations. Biology and Fertility of Soils, v.37, p.237-244, 2003. DOI: 10.1007/s00374-003-0587-4.

BRIEDIS, C.; SÁ, J.C. de M.; DE-CARLI, R.S.; ANTUNES, E.A.P.; SIMON, L.; ROMKO, M.L.; ELIAS, L.S.; FERREIRA, A. de O. Particulate soil organic carbon and stratification ratio increases in response to crop residue decomposition under no-till. Revista Brasileira de Ciência do Solo, v.36, p.1483-1490, 2012. DOI: $10.1590 /$ S0100-06832012000500012.

CAMBARDELlA, C.A.; ELLIOTT, E.T. Particulate soil organic-matter changes across a grassland cultivation sequence. Soil Science Society of America Journal, v.56, p.777-783, 1992. DOI: $10.2136 /$ sssaj1992.03615995005600030017x.
CARVALHO, A.M. de; BUSTAMANTE, M.M. da C.; SOUSA JUNIOR, J.G. de A.; VIVALDI, L.J. Decomposição de resíduos vegetais em latossolo sob cultivo de milho e plantas de cobertura. Revista Brasileira de Ciência do Solo, v.32, p.2831-2838, 2008. DOI: $10.1590 / \mathrm{S} 0100-06832008000700029$.

CARVALHO, A.M. de; MARCHÃO, R.L.; BUSTAMANTE, M.M. da C.; ALCÂNTARA, F.A. de; COSER, T.R. Characterization of cover crops by NMR spectroscopy: impacts on soil carbon, nitrogen and phosphorus under tillage regimes. Revista Ciência Agronômica, v.45, p.968-975, 2014. DOI: 10.1590/ S1806-66902014000500007.

CONCEIÇÃO, P.C.; DIECKOW, J.; BAYER, C. Combined role of no-tillage and cropping systems in soil carbon stocks and stabilization. Soil and Tillage Research, v.129, p.40-47, 2013. DOI: 10.1016/j.still.2013.01.006.

CORAZZA, E.J.; SILVA, J.E.; RESCK, D.V.S.; GOMES, A.C. Comportamento de diferentes sistemas de manejo como fonte ou depósito de carbono em relação à vegetação de cerrado. Revista Brasileira de Ciência do Solo, v.23, p.425-432, 1999.

COSTA, F. de S.; BAYER, C.; ALBUQUERQUE, J.A.; FONTOURA, S.M.V. Aumento de matéria orgânica num Latossolo Bruno em plantio direto. Ciência Rural, v.34, p.587-589, 2004. DOI: $10.1590 /$ S0103-84782004000200041.

DALENBERG, J.W.; JAGER, G. Priming effect of some organic additions to ${ }^{14} \mathrm{C}$-labeled soil. Soil Biology and Biochemistry, v.21, p.443-448, 1989. DOI: 10.1016/0038-0717(89)90157-0.

DICK, D.P.; NOVOTNY, E.H.; DIECKOW, J.; BAYER, C. Química da matéria orgânica do solo. In: MELO, V. de F.; ALLEONI, L.R.F. (Ed.). Química e mineralogia do solo: parte II - aplicações. Viçosa: Sociedade Brasileira de Ciência do Solo, 2009. p.1-68.

DIECKOW, J.; MIELNICZUK, J.; KNICKER, H.; BAYER, C.; DICK, D.P.; KÖGEL-KNABNER, I. Composition of organic matter in a subtropical Acrisol as influenced by land use, cropping and $\mathrm{N}$ fertilization, assessed by CPMAS ${ }^{13} \mathrm{C}$ NMR spectroscopy. European Journal of Soil Science, v.56, p.705-715, 2005. DOI: 10.1111/j.1365-2389.2005.00705.x.

FONTAINE, S.; BAROT, S.; BARRÉ, P.; BDIOUI, N.; MARY, B.; RUMPEL, C. Stability of organic carbon in deep soil layers controlled by fresh carbon supply. Nature, v.450, p.277-280, 2007. DOI: 10.1038 /nature06275.

FONTAINE, S.; MARIOTTI, A.; ABBADIE, L. The priming effect of organic matter: a question of microbial competition? Soil Biology and Biochemistry, v.35, p.837-843, 2003. DOI: 10.1016/ S0038-0717(03)00123-8.

FONTANA, A.; PEREIRA, M.G.; LOSS, A.; CUNHA, T.J.F.; SALTON, J.C. Atributos de fertilidade e frações húmicas de um Latossolo Vermelho no Cerrado. Pesquisa Agropecuária Brasileira, v.41, p.847-853, 2006. DOI: 10.1590/ S0100-204X2006000500018.

GOLCHIN, A.; OADES, J.M.; SKJEMSTAD, J.O.; CLARKE, P. Study of free and occluded particulate organic-matter in soils by solid-state ${ }^{13} \mathrm{C} \mathrm{Cp} / \mathrm{MAS}$ NMR spectroscopy and scanning electron microscopy. Australian Journal of Soil Research, v.32, p.285-309, 1994. DOI: 10.1071/SR9940285. 
GONÇALVES, C.N.; CERETTA, C.A. Plantas de cobertura de solo antecedendo o milho e seu efeito sobre o carbono orgânico do solo, sob plantio direto. Revista Brasileira de Ciência do Solo, v.23, p.307-313, 1999. DOI: 10.1590/S0100-06831999000200015.

KUZYAKOV, Y. Priming effects: interactions between living and dead organic matter. Soil Biology and Biochemistry, v.42, p.1363-1371, 2010. DOI: 10.1016/j.soilbio.2010.04.003.

KUZYAKOV, Y.; FRIEDEL, J.K.; STAHR, K. Review and quantification of priming effects. Soil Biology and Biochemistry, v.32, p.1485-1498, 2000. DOI: 10.1016/S0038-0717(00)00084-5.

LAL, R. Soil carbon sequestration impacts on global climate change and food security. Science, v.304, p.1623-1626, 2004. DOI: 10.1126/science.1097396.

PIVA, J.T.; DIECKOW, J.; BAYER, C.; ZANATTA, J.A.; MORAES, A. de; PAULETTI, V.; TOMAZI, M.; PERGHER, M. No-till reduces global warming potential in a subtropical Ferralsol. Plant and Soil, v.361, p.359-373, 2012. DOI: 10.1007/ s11104-012-1244-1.

REIS, C.E.S. dos; DICK, D.P.; CALDAS, J. da S.; BAYER, C. Carbon sequestration in clay and silt fractions of Brazilian soils under conventional and no-tillage systems. Scientia Agricola, v.71, p.292-301, 2014. DOI: 10.1590/0103-9016-2013-0234.

SÁ, J.C. de M.; LAL, R. Stratification ratio of soil organic matter pools as an indicator of carbon sequestration in a tillage chronosequence on a Brazilian Oxisol. Soil and Tillage Research, v.103, p.46-56, 2009. DOI: 10.1016/j.still.2008.09.003.

SALVO, L.; HERNÁNDEZ, J.; ERNST, O. Distribution of soil organic carbon in different size fractions, under pasture and crop rotations with conventional tillage and no-till systems. Soil and Tillage Research, v.109, p.116-122, 2010. DOI: 10.1016/j. still.2010.05.008.

SOUZA, W.J.O.; MELO, W.J. Matéria orgânica em um Latossolo submetido a diferentes sistemas de produção de milho. Revista Brasileira de Ciência do Solo, v.27, p.1113-1122, 2003. DOI: 10.1590/S0100-06832003000600016.

WENDLING, B.; JUCKSCH, I.; MENDONÇA, E. de S.; NEVES, J.C.L. Carbono orgânico e estabilidade de agregados de um Latossolo Vermelho sob diferentes manejos. Pesquisa Agropecuária Brasileira, v.40, p.487-494, 2005. DOI: 10.1590/ S0100-204X2005000500010.

Recebido em 23 de março de 2015 e aprovado em 19 de novembro de 2015 